\title{
Integrating Course Material and Application: A Progressive Writing Assignment Applied to an Astrochemistry Tutorial
}

\author{
Olivia H. Wilkins* Camillus F. Buzard ${ }^{\dagger}$
}

February 20, 2018

\begin{abstract}
A major challenge in teaching is helping students integrate course concepts to understand the big picture of a field and apply those concepts in new situations. This can be difficult because such mastery is hard for students when they do not understand how to use the knowledge gained. To address this challenge in a tutorial course about astrochemistry (taught by graduate students to chemistry undergraduates), we implemented a progressive writing assignment that culminated in a final presentation. In the progressive writing assignment, students chose an astrochemistry topic they found interesting to be the subject of three sequential papers, which became the basis for their presentations. The purpose of this assignment was to gradually introduce chemistry students to research areas in astronomy, which is by nature outside the general chemistry curriculum, while also providing students with regular feedback. Over the course of the assignment, students applied key themes in the course - significance of astrochemistry research, research methods, and chemistry in astronomical environments - separately to their chosen topics before explaining in the final presentation how these different aspects of astrochemistry work together. We also observed during the final presentations that students seemed to have achieved a deep understanding of their individual topics, perhaps because their papers had required that they had learned the topics well enough to teach to a non-expert reader. By incorporating stories and anaologies, rather than just facts, students gave presentations that were accessible to a novice audience. As a result, students explained broader impacts of astrochemistry research, rather than just focusing on results, and they entertained questions with answers that went beyond clarification of the material discussed.
\end{abstract}

\footnotetext{
*Department of Chemistry \& Chemical Engineering, California Institute of Technology. owilkins@caltech.edu (Corresponding author). Copyright 2018 Olivia H. Wilkins and Camillus F. Buzard. This work is licensed under a Creative Commons Attribution-NonCommercial 4.0 International License (http://creativecommons.org/licenses/by-nc/4.0/).

${ }^{\dagger}$ Department of Chemistry \& Chemical Engineering, California Institute of Technology.

${ }^{\ddagger}$ Submitted 6/23/2017; Accepted, 9/9/2017.
} 
Chemistry tutorials (Ch 101) ${ }^{1}$ were introduced at the California Institute of Technology (Caltech) in Spring 2017. These tutorials are three-unit (as opposed to full nine-unit) special topics courses offered to undergraduates and are taught by graduate student and postdoctoral instructors with guidance from experienced faculty members. Because $\mathrm{Ch}$ 101 is designated as having three units, these classes typically meet 1 hours with 2 hours of work outside of class each of the nine weeks of the spring term. In addition to counting as elective credit for undergrads majoring in chemistry, Ch 101 is an opportunity for students to explore new fields of chemistry or topics relevant to their summer research projects. Our course, Astrochemistry: Spectroscopy in Space, was evenly spread across class year, from first-year to senior undergrads. We first introduced the class to basic astronomy terms and chemistry from an astronomer's perspective before teaching students about the methods used in the field and going over several research areas in the field, drawing from our own research experiences.

A critical component of teaching is facilitating student mastery of course material - that is, not only helping students acquire skills, but also teaching them how to integrate and when to apply them. However, it is often challenging for instructors (the experts) to help their students (the novices) master a subject. One reason for this is that people generally have difficulty focusing on multiple ideas simultaneously, especially in areas not yet mastered. In other words, connecting different concepts in a subject to understand the larger picture is more difficult when individual ideas are not well-understood on their own. Another reason for the difficulty in facilitating mastery is that students often acquire knowledge in a context different from how that knowledge should be applied (Ambrose, Bridges, DiPietro, Lovett, \& Norman, 2010, Ch. 4). For our Ch 101 tutorial, this was especially true because we could not give students hands-on research-type experience, nor did we have time to introduce students to much of the material required for completing traditional problem sets because the course only met for one-third of the time allotted to full courses over a nine-week term. Because our Ch 101 tutorial met only one hour weekly, we were faced with the challenge of showing the breadth of chemistry research in astronomy-related fields with very little time to do so. While we gave an introduction to several areas of research in our lectures, there were many more that were left out. With these challenges in mind, our learning outcomes for the course were that:

1. students would understand the significance of astrochemistry research, learn to read and review literature within the field, and connect different research findings, for instance those from laboratory astrophysics and observational astronomy, and

2. students would become familiar with the breadth of astrochemistry research and some of the techniques by which it is conducted.

In order to help our students achieve the learning outcomes we set, while also addressing the challenges of limited time and helping students connect course material with applications, we designed a progressive writing assignment for our astrochemistry tutorial, inspired by Vollmer (2017), who has used a similar assignment for microbiology. The original microbiology assignment was used for a full-credit course in which students wrote 
a series of one-page (double-spaced) papers about an "adopted" bacterium in the context of a biweekly course theme. After each paper was submitted, the students were asked to share one or two sentences in class about their bacterium (Vollmer, 2016). In contrast, our progressive writing assignment consisted of three short (1-3 pages, single-spaced) papers (as compared to about 7 in the original microbiology assignment) about student-chosen topics related to astrochemistry. The individual papers were submitted two weeks apart and ended up fitting together to create a longer cohesive essay that was also the basis of final 10-minute presentations.

Each of the three papers in our assignment focused on a different theme - significance and history, methods, and chemical findings - to guide students to think about different aspects of their chosen topics and write for a reader with little astronomy background. Each paper was expected to be written for the same beginner audience, with the caveat the audience would have read each student's previous papers. For the first paper in the assignment (significance and history), we asked students to introduce their topic to the reader by providing a brief history of the topic and explaining its scientific and cultural significance. As an example, "Mysha," who chose the chemistry of binary stars as her topic, evoked a familiar pop culture reference as an introduction to the history and significance of binary stars: Luke Skywalker's home planet of Tatooine, which orbits a pair of binary stars in the movie Star Wars. The second paper was intended not only to describe methods used for studying the topic chosen for the assignment but to also discuss the benefits and challenges of different methods in the field. Mysha accomplished this by comparing and contrasting three methods for observing binary stars, illustrating the methods with figures and incorporating plots that demonstrated what binary stars look like to the astronomers studying them. For the final paper, students were asked to incorporate chemistry into their progressive writing assignment by highlighting their topic's chemistry generally and explaining how that chemistry contributes to our understanding of the wider universe. In Mysha's final paper, she explained the connection between binary stars' chemical composition and where they formed. She also gave examples of stars in binary systems with different compositions from their companion stars, which helped reveal that even if stars form within the same region of space, they can have significant chemical differences. At the end of the term, students were asked to assemble the information from their three papers into a talk to present to their classmates.

Using this assignment, we approached the first goal of enabling students to draw connections between different areas of astrochemistry research using both the format of the prompt itself as well as the timing of the individual short papers in the assignment. The format of the assignment follows the guidelines for transparent teaching methods ${ }^{2}$ demonstrated by Winkelmes (2017). Transparency in assignments - which helps students to understand how and why they are learning by defining learning benefits such as skills practiced, knowledge gained, and criteria for success - has been linked to students' having greater academic confidence and academic success (Gianoutsos \& Winkelmes, 2016; Winkelmes et al., 2016). For educational writing specifically, higher levels of confidence 
in writing ability have been found to correlate with more success in completing writing tasks (Ekholm, Zumbrunn, \& Conklin, 2015; Jones, 2008; Pajares, 2003).

Because our Ch 101 students had little-to-no prior knowledge in the course subject and had previous writing experiences that were likely dominated by more technical writing (whereas we were asking for a more popular science style of writing), we felt a transparentlywritten assignment was important to both help students connect astrochemistry concepts in the context of their chosen topics as well as to minimize stress from not knowing our expectations for the individual papers. In the assignment prompt, we presented assignment outcomes (including skills being practiced) and listed our expectations for the papers (consulting a variety of references, connecting the topic to concepts from lecture material, and explaining the significance of scientific findings). For each paper, we also included a list of guiding questions and statements to help students think about their topic in the context of the paper's theme. For instance, under the description for the third paper (chemical findings), we included, "The implications of chemical findings might include understanding star formation better or identifying a [molecule] formation reaction scheme," to give students an idea of how they might connect chemical findings to their topic's significance, which was the subject of the first paper.

The spacing of deadlines within the assignment also enabled students to make connections between course material and chosen topics. Our lectures were divided into units such that they loosely matched the themes of the short papers, and each paper was due a week after the conclusion of the related unit. The outline of lecture units and corresponding paper themes is shown in Table 1.

Table 1: Showing the correspondence between lecture topics and short paper themes.

\begin{tabular}{cc}
\hline Lecture topics & Paper theme \\
\hline Introduction to astrochemistry & \\
Chemistry laboratories in space & Significance and history \\
Spectroscopy & \\
Observational astrochemistry techniques / Telescopes \\
Physics and chemistry of the interstellar medium \\
Organic chemistry in space \\
Exoplanet origins
\end{tabular}

By having lectures in parallel with the themes of the papers, we were able to give students some basic knowledge of the field generally and demonstrate the level at which we expected their papers to be written (to an audience with a general chemistry background but no astronomy background). Students, most of whom had little or no previous astronomy background, could focus on different components of their topics (i.e. describing 
an environment in space, explaining the merits of the research field, observational techniques) individually before considering them together in the final presentation. By immersing themselves in their individual topics, students could more easily understand how course concepts applied to application. Furthermore, by having students submit three papers over the course of the term rather than one final paper, students were regularly given feedback they could then implement into subsequent papers. By their final presentations, students had been given three rounds of feedback from both instructors. ${ }^{3}$

The second goal of exposing students to the breadth of astrochemistry research was achieved by having the students hear one another's final presentations to the class about their respective astrochemistry topic. Specifically, while we discussed organic chemistry in the interstellar medium and exoplanet formation, students also heard from their peers about the chemistry of binary stars, cometary ices, protoplanetary disks, Titan (one of Saturn's moons), Venus's atmosphere, and brown dwarfs.

Students responded positively to the progressive writing assignment. In an anonymous end-of-term survey, we asked the six students, "How did you find the assignment/project?" with a list of 16 adjectives ranging from negative (e.g. scary, awful) to positive (e.g. helpful, fun). The responses selected by students are shown in Table 2. Largely positive adjectives were selected, and "interesting" was selected by all students. Several students also commented that they liked being able to focus the assignment on a topic that interested them. Such comments included, "I definitely learned from putting together the papers," and "[Assignments were] quite fun because of the freedom of topics." One student even expressed appreciation for honing communication skills, writing, "[I liked that the assignment] forced me to practice writing and presenting."

Table 2: Adjectives selected by Ch 101 students in response to "How did you find the assignment/project?"

The following options were given but not selected by any of the students: scary, awful, boring, weird, challenging, frustrating, different, confusing, tedious, other (with room to explain).

\begin{tabular}{cc}
\hline Adjective selected & \# responses $(n=6)$ \\
\hline Interesting & 6 \\
Fun & 3 \\
Enlightening & 2 \\
Difficult & 1 \\
Helpful & 1 \\
Great & 1 \\
Okay & 1 \\
\hline
\end{tabular}


We, as the instructors, were pleased with how each student implemented elements of storytelling by combining scientific results with commentary on their topic's history and significance. We noticed that there was substantial variation in how students approached telling the story of their topics. Some students wrote their papers as if they were long introductions to a journal article, explaining jargon but remaining formal, while others were more illustrative, prompting the reader to imagine and connect to their topic emotionally. Despite these differences, students across this spectrum effectively justified why their topic was important, citing examples of astrobiology, planetary evolution, and understanding how formation environment affects chemical characteristics. In future use of this assignment, we might encourage students to write in a more standardized style, for instance that of a Science article.

At the end of the course, we asked students to estimate how long it took them to complete individual papers. Because papers were due every two weeks of the three-unit course (meaning about 1 hour in class and 2 hours of work out of class per week), our goal was that students would require about 4 hours total per paper. ${ }^{4}$ There was variability in students' responses for all papers. For Paper I, there was an even distribution of reported time from 1-2 hours to 6 hours, with 4 hours being the median. Students required much more time for Paper II, with two students reporting it took them 4 hours, one reporting 5 hours, and three reporting the assignment required 6 hours. Finally, four (out of six) students reported the final paper required 4 hours to complete and two reported that they needed 6 hours. Because students did not require more than 6 hours to complete the individual papers in the progressive writing assignment overall (and because we received no complaints about the assignment taking too much time), we find our expectations for the progressive writing assignment to be reasonable. However, Paper II stood out as taking students longer, so if we implement this assignment again, we will reconsider how we teach astrochemistry methods in class $^{5}$ and present expectations for the paper.

In the end-of-course survey, half of the students commented on what they would change. One student commented that they "thought the assignment was going to be a paper that became one larger paper" and that they would have preferred if each new paper included revised versions of the previous paper(s). Another student wrote, "The only thing I would prefer more is if there weren't any papers at all, but that's not reasonable." One student indicated they felt Paper I (significance and history) was not as interesting for their topic as it was for other students' topics. In the future, we may try to explicitly discuss our intentions and expectations for the papers in the first class, so that students fully understand what we are looking for from each of the papers and why we think the writing experience will be useful to them as science students. For instance, we might explain that we had chosen to not have the three papers be extensions or revisions of each other because we wanted the students to approach their chosen topics exclusively from different themes throughout the course of term, while using our feedback to improve the style and tone of their future papers. Moreover, we would explain that the final presentation would give them the opportunity to revisit the information they learned for each paper and incorporate our comments. We would also more clearly establish 
that each paper in the assignment is meant to be part of a larger document covering individual student topics.

In the first paper, we found that many students were having difficulty pinpointing their target audience, which was meant to be a student (perhaps someone else in the class) who knew at least some basic chemistry but had limited or no prior knowledge about astronomy. Some students either did not realize that they were including jargon unfamiliar to their audience or were having trouble understanding some concepts themselves. After we pointed out these instances in our feedback for the first paper, however, students improved immensely in targeting their audience in the second and third papers. We may try to address the problem in the future by providing example essays. Furthermore, some students effectively used figures to help illustrate concepts in their papers, but others did not use figures at all. Students who did not use figures generally struggled to clearly describe various processes and characteristics about their topic more than those who used figures. If we use this assignment in the future, we will likely require at least one relevant figure per paper.

The final presentations were intended to be based on the progressive writing assignment. While this is the first class we have taught at Caltech, we can compare the quality of these presentations to ones we have seen in three other contexts: in courses we have taken at Caltech, in courses we have taken at our own undergraduate institutions (both liberal arts colleges), and at conferences. The most straightforward comparison to make is to presentations from other Caltech courses, keeping in mind that some differences in presentation quality may result from Ch 101 being an introductory course for undergraduates and courses we have taken being geared for upper-level undergraduates and beginning graduate students. Nonetheless, we found that our students' talks were more informative and cohesive than many upper-level course presentations we have observed. We think that this can be attributed to both the students' involvement with their respective topics over practically the whole term and the opportunities they had to incorporate our feedback on their papers into their presentations. We also felt that by explicitly having our students write for a novice audience, we put them in a position to more deeply understand and teach about their topics rather than just relay facts and assume their audience had advanced prior knowledge.

In their final presentations, the students all introduced their topics to the class at a novice level and explained the merit of scientific research about each of their topics. Both of these were requirements of the first paper but neither has been universally given in student presentations in our experiences. In both the assignment prompt and in our feedback, we emphasized these aspects of the project, and we saw improvements in how students targeted their audience from the first paper to the final presentation. For instance, Mysha had introduced the concept of a Roche Lobe in her introductory paper on binary stars. In our comments, we had suggested that this more advanced topic might require more explanation, especially to a novice audience. When she presented the same concept in her talk, she explained it in her own words, making it clear she had taken the time to go back and really understand the material. The repetitive nature of our 
progressive writing assignment, and the opportunity it gave students to incorporate our feedback, encouraged them to gain a deeper understanding of their given topic than a traditional final presentation would have.

The students also generally shared the science of their topic within the framework of its history, effectively telling its story in a more interesting way than merely reading off scientific data. Again, this was a requirement of the progressive writing assignment. In addition to the benefit of having an initial exposure to this way of scientific storytelling, we think that the length of time devoted to studying the same topic, and the nature of the progressive writing assignment, allowed the students to become more invested in their topics and begin to see them as cohesive stories rather than as collections of facts. An example that illustrates this is the way "Aarish" answered a question about limitations of the instruments used to study Venus's atmosphere. Rather than just answering with a quick fact, he enthusiastically told the class about several probes that made it to the surface of Venus but could not take pictures; in one case, the camera lens caps simply failed to release, while in another the probe tested the compressibility of an ejected lens cap rather than that of Venus's surface. These types of answers more closely resemble those given by experts at conferences - in their investment in the topic and excitement to share more details than they had time for in their talks - than those given in typical class presentations.

Overall, it seemed that students were interested with the topics through lenses from history to scientific findings, perhaps because they had been invested in these projects throughout the duration of the course and had mastered not only astrochemistry of a specific topic but also how to integrate thinking about a topic's history, cultural and scientific significance, methods, and chemical research. This engagement appeared to translate to the students' asking questions after each presentation; despite grumbling at our request for students to ask questions, all students - even students who did not normally participate in class discussion — asked multiple questions about each other's topics.

In addition to the technical improvements we saw in the quality of the students' presentations as compared to presentations typically given in undergraduate courses, we think the format of our assignment allowed for a less stressful learning experience for the students than the typical final presentation in which the bulk of the research and assembling of the talk is crammed into the last few weeks of the term. We consider this alleviation of the typical stress to be an important criterion in judging an assignment's quality. Our assignment required students to spread out their work almost evenly throughout the term. It ensured that they could not wait until the last minute and cram, thereby allowing them to produce better work without the stress of an imminent deadline. For these reasons, we think that our progressive writing assignment resulted in student presentations that were better than those commonly given in an undergraduate course.

As a result of adapting the progressive writing assignment presented by Vollmer (2017) to our Ch 101 astrochemistry tutorial, we achieved our goals of enabling students to connect 
course material directly to an application of that material and of exposing students to a wider range of topics than we could address in the lectures. Students responded positively to the assignment overall, and from their feedback and our own assessment of their assignments and final presentations, we observe that they successfully learned to integrate different components of astrochemistry (i.e. basic astronomy, observational and analytical methods, and chemical findings). Furthermore, students appeared engaged and invested in the course and especially in the project, despite the course counting for only one-third of a full course. We would recommend such an assignment in introductory-type (and specifically tutorial) courses in which students are learning material outside their typical curriculum at a rapid pace.

\section{The assignment: Ch 101 - Astrochemistry, Progressive Writing Assignment}

See the Supplementary Files for this article at thepromptjournal.com for a PDF facsimile of the original formatting of this assignment.

Distilled objective: To research, apply lecture topics to, and compile the story of a chosen region or class of bodies in space of both astrochemical and personal interest.

Skills:

- Identifying key concepts in scientific literature

- Storytelling as an effective science communication tool

- Analyzing connections among research methods, instrumentation, study subject, and results

- Writing reviews

Outcomes: By the end of this assignment, you will have practiced the aforementioned skills as well as have compiled a booklet of information about a specific astrochemical topic. This progressive writing assignment will also serve as the basis of research for the final presentation.

Submission: All assignments should be submitted via email to the instructors by 5:00 p.m. on Friday of the week specified in the timeline below. The subject line of submissions should follow the format "Ch 101 - [assignment]" where [assignment] = paper I, paper II, paper III.

Assignment: Astrochemistry, though young, is studied in a broad variety of environments and with many different techniques. We will not have time to cover numerous interesting facets of the field in this course. Therefore, the progressive writing assignment is designed to help students investigate, and eventually teach (in a 10-to-15-minute presentation), topics not covered in class. 
Over the course of the term, you will write three short (1-to-3-page) single-spaced papers about an approved topic: a region of space, class of celestial bodies, or other area of astrochemistry research that is both astrochemically and personally interesting. Appropriate topics include, but are not limited to, a specific molecular gas cloud, comets, and protoplanetary disks. A more complete list of possible topics is included below.

Throughout this assignment, you should:

- consult a variety of references - lecture materials, academic literature, textbooks, etc.- - analyzing your sources for key concepts to be included in a review of the research and history of your topic.

- connect ideas among research methods, instrumentation, study subject, and results, in effect connecting the concepts from lecture with an area of research.

- analyze the significance of scientific findings, explaining how they have made an impact on the wider astrochemistry and scientific communities.

- not only survey scientific findings but tell the story of your subject, incorporating history and secondary sources (e.g. news articles) where appropriate.

The themes of the papers are [I] significance and history, [II] methods, and [III] chemical findings.

I. Significance and history. This first paper will introduce your topic and should address the importance of your topic to both astrochemistry specifically and to science generally. Introduce your topic assuming your reader may have little astronomy background (they know that stars exist but perhaps not much else). Then, describe the implications of studying your topic, i.e. why should we care? (Does it have astrobiological significance? Is it the site of a major discovery? Is it particularly molecule-rich or -poor?) You should also give a brief history of your topic, discussing its discovery, previous historical niche (e.g. before pulsars were understood, their radio signals were labeled "LGM" for "little green men'), key players and discoveries throughout history, and the current state of knowledge (what are the big questions/triumphs that define your topic?).

II. Methods. In the second paper, address methods used (direct sampling, spectroscopy), instruments (both generally, e.g. single-dish radio telescopes, and specifically, e.g. the GBT), and data analysis (types of data, analysis tools, etc.). You should also discuss the reasoning behind the methods used. (For instance, if strictly qualitative data such as molecular identification is used without quantitative data such as abundance calculations, explain why. Are there complications that arise in making such calculations, such as poor resolution or missing information? Or is the emphasis on probing your target as a preliminary survey for later studies?)

III. Chemical findings. The final paper should discuss chemical findings broadly, identifying specific molecules detected, abundances (relative or absolute), and specific implications. The implications of chemical findings might include understanding star formation better 
or identifying a formation reaction scheme. You should also assess chemical characteristics of your topic, identifying - for instance - whether it is particularly oxygen-rich, populated by unsaturated carbon chains, or without any defining characteristics/diverse populations.

\section{Deadlines:}

Week 2 Submit topic idea for approval by instructors (by Friday, 5 p.m.)

Week 3 Paper I due (Friday, 5 p.m.)

Week 5 Paper II due (Friday, 5 p.m.)

Week 7 Paper III due (Friday, 5 p.m.)

Week 9 Final presentation

\section{Possible topic ideas:}

- Orion molecular cloud

- Cometary ices

- Galaxies (21-cm, rotation, total mass, dark matter)

- Brown dwarfs (young versus field, $\mathrm{CO} / \mathrm{CH}_{4}$ )

- Theory/modeling

- Astrobiology

- Atmospheric chemistry

- Protoplanetary disks

- Ice chemistry

- Isotope/isomer/other chemical ratios

Acknowledgments: The authors thank their Ch 101 faculty advisor Dr. Mitchio Okumura and the Center for Teaching, Learning, and Outreach at Caltech for their support and suggestions. This work benefited from discussions with Dr. Jennifer E. Weaver about teaching pedagogy. The authors also thank the referees for their helpful comments.

\section{Notes}

${ }^{1}$ At Caltech, courses numbered 0-100 are undergraduate courses and courses numbered 100 and above are typically taken by graduate students and advanced undergraduates.

${ }^{2} \mathrm{~A}$ list of transparent methods, including examples, is provided by the Transparency in Learning and Teaching Project at the University of Nevada, Las Vegas at https://www.unlv.edu/provost/transparency

${ }^{3}$ Some students also took advantage of opportunities to receive feedback by email or in office hours. 


\footnotetext{
${ }^{4}$ At the beginning of the term, we introduced the students to the SAO/NASA Astrophysics Data System (http://adsabs.harvard.edu), an online catalog of astrophysics and physics research literature. We assumed that they had previous experience working with such databases. However, an introduction to online research databases could be given at the beginning of the course, especially for a class where students could not reasonably be assumed to have previous experience searching for research literature.

${ }^{5}$ Lectures about methods focused on spectroscopy and radio telescopes, which are common tools in astrochemistry. However, for many of the topics that students ended up choosing, radio telescopes are generally not used.
}

\section{References}

Ambrose, S. A., Bridges, M. W., DiPietro, M., Lovett, M. C., \& Norman, M. K. (2010). How learning works: Seven research-based principles for smart teaching. San Francisco: Jossey-Bass.

Ekholm, E., Zumbrunn, S., \& Conklin, S. (2015). The relation of college student selfefficacy toward writing and writing self-regulation aptitude: Writing feedback perceptions as a mediating variable. Teaching in Higher Education, 20(2), 197-207. http://doi.org/ 10.1080/13562517.2014.974026

Gianoutsos, D., \& Winkelmes, M. A. (2016). Navigating with transparency. In Proceedings of the Pennsylvania Association of Developmental Educators.

Jones, E. (2008). Predicting performance in first-semester college basic writers: Revisiting the role of self-beliefs. Contemporary Educational Psychology, 33(2), 209-238. http: //doi.org/10.1016/j.cedpsych.2006.11.001

Pajares, F. (2003). Self-efficacy beliefs, motivation, and achievement in writing: A review of the literature. Reading \& Writing Quarterly, 19(2), 139-158. http://doi.org/10.1080/ 10573560390143085

Vollmer, A. (2016, November 16). "Adopting" a pathogen. Retrieved from http:// schaechter.asmblog.org/schaechter/2016/09/adopting-a-pathogen.html

Vollmer, A. (2017). Your top 10 teaching headaches and how to turn them into learning opportunities. Seminar presented at the Caltech Project for Effective Teaching Winter Seminar Series, Pasadena, CA.

Winkelmes, M. A. (2017, January). Teaching with transparency: Empowering equitable learning. Seminar and Workshop presented at the Caltech TeachWeek, Pasadena, CA.

Winkelmes, M. A., Bernacki, M., Butler, J., Zochowski, M., Golanics, J., \& Weavil, K. H. (2016). A teaching intervention that increases underserved college students success. Peer Review, 18(1/2). Retrieved from https://www.aacu.org/peerreview/2016/winterspring/ Winkelmes 